\title{
Exact Traveling Wave Solutions for the System of Shallow Water Wave Equations and Modified Liouville Equation Using Extended Jacobian Elliptic Function Expansion Method
}

\author{
Emad H. M. Zahran1, Mostafa M. A. Khater ${ }^{2 *}$ \\ ${ }^{1}$ Department of Mathematical and Physical Engineering, College of Engineering, University of Benha, Shubra, \\ Egypt \\ ${ }^{2}$ Department of Mathematics, Faculty of Science, Mansoura University, Mansoura, Egypt \\ Email: mostafa.Khater2024@yahoo.com
}

Received 11 September 2014; revised 21 October 2014; accepted 15 November 2014

Copyright @ 2014 by authors and Scientific Research Publishing Inc.

This work is licensed under the Creative Commons Attribution International License (CC BY).

http://creativecommons.org/licenses/by/4.0/

\section{(c) (i) Open Access}

\begin{abstract}
In this work, an extended Jacobian elliptic function expansion method is proposed for constructing the exact solutions of nonlinear evolution equations. The validity and reliability of the method are tested by its applications to the system of shallow water wave equations and modified Liouville equation which play an important role in mathematical physics.
\end{abstract}

\section{Keywords}

Extended Jacobian Elliptic Function Expansion Method, The System of Shallow Water Wave Equations, Modified Liouville Equation, Traveling Wave Solutions, Solitary Wave Solutions

\section{Introduction}

The nonlinear partial differential equations of mathematical physics are major subjects in physical science [1]. Exact solutions for these equations play an important role in many phenomena in physics such as fluid mechanics, hydrodynamics, optics, plasma physics and so on. Recently many new approaches for finding these solu-

"Corresponding author. 
tions have been proposed, for example, tanh-sech method [2]-[4], extended tanh-method [5]-[7], sine-cosine method [8]-[10], homogeneous balance method [11] [12], F-expansion method [13]-[15], exp-function method [16], the modified simple equation method [17], the $\exp (-\phi(\xi))$-expansion method [18], $\left(\frac{G^{\prime}}{G}\right)$-expansion method [19]-[22], Jacobi elliptic function method [23]-[26] and so on.

The objective of this article is to apply the extended Jacobian elliptic function expansion method for finding the exact traveling wave solution the system of shallow water wave equations and modified Liouville equation which play an important role in mathematical physics.

The rest of this paper is organized as follows: In Section 2, we give the description of the extended Jacobi elliptic function expansion method. In Section 3, we use this method to find the exact solutions of the nonlinear evolution equations pointed out above. In Section 4, conclusions are given.

\section{Description of Method}

Consider the following nonlinear evolution equation

$$
F\left(u, u_{t}, u_{x}, u_{t t}, u_{x x}, \cdots\right)=0,
$$

where $F$ is polynomial in $u(x, t)$ and its partial derivatives in which the highest order derivatives and nonlinear terms are involved. In the following, we give the main steps of this method [23]-[26].

Step 1. Using the transformation

$$
u=u(\xi), \xi=x-c t,
$$

where $c$ is wave speed, to reduce Equation (1) to the following ODE:

$$
P\left(u, u^{\prime}, u^{\prime \prime}, u^{\prime \prime \prime}, \cdots\right)=0 \text {, }
$$

where $P$ is a polynomial in $u(\xi)$ and its total derivatives, while ' $=\frac{\mathrm{d}}{\mathrm{d} \xi}$ '.

Step 2. Making good use of ten Jacobian elliptic functions, we assume that (3) has the solutions in these forms:

$$
u(\xi)=a_{0}+\sum_{j=1}^{N} f_{i}^{j-1}(\xi)\left[a_{j} f_{i}(\xi)+b_{j} g_{i}(\xi)\right], i=1,2,3, \cdots,
$$

with

$$
\begin{array}{ll}
f_{1}(\xi)=s n \xi, & g_{1}(\xi)=c n \xi, \\
f_{2}(\xi)=s n \xi, & g_{2}(\xi)=d n \xi, \\
f_{3}(\xi)=n s \xi, & g_{3}(\xi)=c s \xi, \\
f_{4}(\xi)=n s \xi, & g_{4}(\xi)=d s \xi, \\
f_{5}(\xi)=s c \xi, & g_{5}(\xi)=n c \xi, \\
f_{6}(\xi)=s d \xi, & g_{6}(\xi)=n d \xi,
\end{array}
$$

where $s n \xi, c n \xi, d n \xi$, are the Jacobian elliptic sine function, the jacobian elliptic cosine function and the Jacobian elliptic function of the third kind and other Jacobian functions which is denoted by Glaisher's symbols and are generated by these three kinds of functions, namely

$$
\begin{aligned}
& n s \xi=\frac{1}{s n \xi}, n c \xi=\frac{1}{c n \xi}, n d \xi=\frac{1}{d n \xi}, s c \xi=\frac{c n \xi}{s n \xi}, \\
& c s \xi=\frac{s n \xi}{c n \xi}, d s \xi=\frac{d n \xi}{s n \xi}, s d \xi=\frac{s n \xi}{d n \xi},
\end{aligned}
$$

that have the relations

$$
\begin{aligned}
& s n^{2} \xi+c n^{2} \xi=1, d n^{2} \xi+m^{2} s n^{2} \xi=1, n s^{2} \xi=1+c s^{2} \xi, \\
& n s^{2} \xi=m^{2}+d s^{2} \xi, s c^{2} \xi+1=n c^{2} \xi, m^{2} s d^{2}+1=n d^{2} \xi,
\end{aligned}
$$


with the modulus $m(0<m<1)$. In addition we know that

$$
\frac{\mathrm{d}}{\mathrm{d} \xi} s n \xi=c n \xi d n \xi, \frac{\mathrm{d}}{\mathrm{d} \xi} c n \xi=-s n \xi d n \xi, \frac{\mathrm{d}}{\mathrm{d} \xi} d n \xi=-m^{2} \operatorname{sn} \xi c n \xi .
$$

The derivatives of other Jacobian elliptic functions are obtained by using Equation (8). To balance the highest order linear term with nonlinear term we define the degree of $u$ as $D[u]=n$ which gives rise to the degrees of other expressions as

$$
D\left[\frac{\mathrm{d}^{q} u}{\mathrm{~d} \xi^{q}}\right]=n+q, \quad D\left[u^{p}\left(\frac{\mathrm{d}^{q} u}{\mathrm{~d} \xi^{q}}\right)^{s}\right]=n p+s(n+q) .
$$

According the rules, we can balance the highest order linear term and nonlinear term in Equation (3) so that $n$ in Equation (4) can be determined.

Noticed that $\operatorname{sn} \xi \rightarrow \tanh \xi, \quad \mathrm{cn} \xi \rightarrow \operatorname{sech} \xi, d n \xi \rightarrow \operatorname{sech} \xi$ when the modulus $m \rightarrow 1$ and $\operatorname{sn} \xi \rightarrow \sin \xi$, $c n \xi \rightarrow \cos \xi, d n \xi \rightarrow 1$ when the modulus $m \rightarrow 0$, we can obtain the corresponding solitary wave solutions and triangle function solutions, respectively, while when therefore Equation (5) degenerate as the following forms

$$
\begin{gathered}
u(\xi)=a_{0}+\sum_{j=1}^{N} \tanh ^{j-1}(\xi)\left[a_{j} \tanh (\xi)+b_{j} \operatorname{sech}(\xi)\right], \\
u(\xi)=a_{0}+\sum_{j=1}^{N} \operatorname{coth}^{j-1}(\xi)\left[a_{j} \operatorname{coth}(\xi)+b_{j} \operatorname{coth}(\xi)\right], \\
u(\xi)=a_{0}+\sum_{j=1}^{N} \tan ^{j-1}(\xi)\left[a_{j} \tan (\xi)+b_{j} \sec (\xi)\right], \\
u(\xi)=a_{0}+\sum_{j=1}^{N} \cot ^{j-1}(\xi)\left[a_{j} \cot (\xi)+b_{j} \csc (\xi)\right] .
\end{gathered}
$$

Therefore the extended Jacobian elliptic function expansion method is more general than sine-cosine method, the tan-function method and Jacobian elliptic function expansion method.

\section{Application}

\subsection{Example 1: The System of Shallow Water Wave Equations}

We first consider the system of the shallow water wave equation [27] in order to demonstrate the $\exp (-\phi(\xi))$ expansion method

$$
\left\{\begin{array}{l}
u_{t}+(u v)_{x}+v_{x x x}=0 \\
v_{t}+u_{x}+v v_{x}=0
\end{array}\right.
$$

We use the wave transformation $u(x, t)=u(\xi), \xi=x-c t$ to reduce Equations (14) to the following nonlinear system of ordinary differential equations:

$$
\left\{\begin{array}{l}
-c u^{\prime}+v u^{\prime}+u v^{\prime}+v^{\prime \prime \prime}=0, \\
u^{\prime}-c v^{\prime}+v v^{\prime}=0,
\end{array}\right.
$$

where by integrating once the second equation with zero constant of integration, we find

$$
u=c v-\frac{v^{2}}{2}
$$

substituting Equation (16) into the first equation of Equation (15) we obtain

$$
v^{\prime \prime \prime}+\left(3 c v-\frac{3 v^{2}}{2}-c^{2}\right) v^{\prime}=0 .
$$

Integrating Equation (17) with zero constant of integration, we find 


$$
v^{\prime \prime}+\frac{3}{2} c v^{2}-\frac{1}{2} v^{3}-c^{2} v=0 .
$$

Balancing $v^{\prime \prime}$ and $v^{3}$ in Equation (18) yields, $N+2=3 N \Rightarrow N=1$. This suggests the choice of $v(\xi)$ in Equation (18) as

$$
u=a_{0}+a_{1} s n+b_{1} c n
$$

where $a_{0}, a_{1}$ and $b_{1}$ are constant such that $a_{1} \neq 0$ or $b_{1} \neq 0$. From (19), it is easy to see that

$$
u^{\prime}=a_{1} c n d n-b_{1} s n d n,
$$

$$
u^{\prime \prime}=-m^{2} s n a_{1}+2 a_{1} s n^{3} m^{2}+2 m^{2} s n^{2} c n b_{1}-a_{1} s n-b_{1} c n .
$$

Substituting Equations (19) and (21) into Equation (18) and equating all coefficients of $s n^{3}, s n^{2} c n, s n^{2}$, sncn, $s n, c n, s n^{0}$ respectively to zero, we obtain:

$$
\begin{gathered}
2 a_{1} m^{2}-\frac{1}{2} a_{1}^{3}+\frac{3}{2} a_{1} b_{1}^{2}=0, \\
2 m^{2} b_{1}-\frac{3}{2} a_{1}^{2} b_{1}+\frac{1}{2} b_{1}^{3}=0, \\
\frac{3}{2} c a_{1}^{2}-\frac{3}{2} c b_{1}^{2}-\frac{3}{2} a_{0} a_{1}^{2}+\frac{3}{2} a_{0} b_{1}^{2}=0, \\
3 c a_{1} b_{1}-3 a_{0} a_{1} b_{1}=0, \\
-a_{1} m^{2}-a_{1}+3 c a_{0} a_{1}-\frac{3}{2} a_{0}^{2} a_{1}-\frac{3}{2} a_{1} b_{1}^{2}-c^{2} a_{1}=0, \\
-b_{1}+3 c a_{0} b_{1}-\frac{3}{2} a_{0}^{2} b_{1}-\frac{1}{2} b_{1}^{3}-c^{2} b_{1}=0, \\
\frac{3}{2} c\left(a_{0}^{2}+b_{1}^{2}\right)-\frac{1}{2} a_{0}^{3}-\frac{3}{2} a_{0} b_{1}^{2}-c^{2} a_{0}=0 .
\end{gathered}
$$

Solving the above system with the aid of Maple or Mathematica, we have the following solution:

\section{Case 1.}

$$
c=a_{0}= \pm \sqrt{2 m^{2}+2}, a_{1}= \pm 2 m, b_{1}=0 .
$$

So that the solution of Equation (18) can be written as

$$
u= \pm \sqrt{2 m^{2}+2} \pm 2 m s n
$$

when $m=1$, the solution can be in the form

$$
u= \pm 2 \pm 2 \tanh (\xi)
$$

Case 2.

$$
c=a_{0}= \pm \sqrt{2-m^{2}}, a_{1}= \pm m, b_{1}= \pm i m
$$

So that the solution of Equation (18) can be written as

$$
u= \pm \sqrt{2-m^{2}} \pm m s n \pm i m c n
$$

when $m=1$, the solution can be in the form

$$
u= \pm 1 \pm \tanh (\xi) \pm i \operatorname{sech}(\xi) .
$$

Case 3.

$$
c=a_{0}= \pm \sqrt{2-4 m^{2}}, a_{1}=0, b_{1}= \pm 2 i m
$$


So that the solution of Equation (18) can be written as

$$
u= \pm \sqrt{2-4 m^{2}} \pm 2 i m c n,
$$

when $m=1$, the solution can be in the form

$$
u= \pm \sqrt{-2} \pm 2 i \operatorname{sech}(\xi) .
$$

\subsection{Example 2: Modified Liouville Equation}

Now, let us consider the modified Liouville equation [28].

$$
a^{2} u_{x x}-u_{t t}+b \mathrm{e}^{\beta u}=0,
$$

respectively, where $a, \beta$ and $b$ are non zero and arbitrary coefficients. Using the wave transformation $u(x, t)=u(\xi), \quad \xi=k x+\omega t, \quad v=\mathrm{e}^{\beta u}$, to reduce Equation (35) to be in the form:

$$
\left(\frac{k^{2} a^{2}}{\beta}-\frac{\omega^{2}}{\beta}\right) v^{\prime \prime} v-\left(\frac{k^{2} a^{2}}{\beta}-\frac{\omega^{2}}{\beta}\right) v^{\prime 2}+b v^{3}=0 .
$$

Balancing $v^{\prime \prime} v$ and $v^{3}$ in Equation (36) yields, $N+2+N=3 N \Rightarrow N=2$. Consequently, we have the formal solution:

$$
u(\xi)=a_{0}+a_{1} s n+b_{1} c n+a_{2} s n^{2}+b_{2} s n c n,
$$

where $a_{0}, a_{1}, a_{2}$ are constants to be determined, such that $a_{2} \neq 0$ or $b_{2} \neq 0$. It is easy to see that

$$
\begin{gathered}
u^{\prime}=a_{1} c n d n-b_{1} s n d n+2 d n a_{2} s n c n-2 d n b_{2} s n^{2}+d n b_{2}, \\
u^{\prime \prime}=-m^{2} s n a_{1}+2 a_{1} s n^{3} m^{2}+2 m^{2} s n^{2} c n b_{1}-4 a_{2} m^{2} s n^{2}+6 a_{2} s n^{4} m^{2}+6 m^{2} s n^{3} c n b_{2} \\
-m^{2} s n c n b_{2}-a_{1} s n-b_{1} c n+2 a_{2}-4 a_{2} s n^{2}-4 b_{2} s n c n .
\end{gathered}
$$

Substituting (37) and (39) into Equation (36) and equating all the coefficients of $s n^{6}, s n^{5} c n, s n^{5}, s n^{4} c n$, $s n^{4}, s n^{3} c n, s n^{3}, s n^{2} c n, s n^{2}, s n c n, s n, c n, s n^{0}$ to zero, we deduce respectively

$$
\begin{aligned}
& \left(\frac{k^{2} a^{2}}{\beta}-\frac{\omega^{2}}{\beta}\right)\left(-2 m^{2} b_{2}^{2}+2 a_{2}^{2} m^{2}\right)+b\left(a_{2}^{3}-3 a_{2} b_{2}^{2}\right)=0, \\
& 4\left(\frac{k^{2} a^{2}}{\beta}-\frac{\omega^{2}}{\beta}\right) a_{2} m^{2} b_{2}+b\left(3 a_{2}^{2} b_{2}-b_{2}^{3}\right)=0, \\
& \left(\frac{k^{2} a^{2}}{\beta}-\frac{\omega^{2}}{\beta}\right)\left(-4 m^{2} b_{1} b_{2}+4 a_{1} m^{2} a_{2}\right)+b\left(3 a_{1} a_{2}^{2}-6 b_{1} a_{2} b_{2}-3 a_{1} b_{2}^{2}\right)=0, \\
& \left(\frac{k^{2} a^{2}}{\beta}-\frac{\omega^{2}}{\beta}\right)\left(4 a_{1} m^{2} b_{2}+4 m^{2} b_{1} a_{2}\right)+b\left(-3 b_{1} b_{2}^{2}+3 b_{1} a_{2}^{2}+6 a_{1} a_{2} b_{2}\right)=0, \\
& \left(\frac{k^{2} a^{2}}{\beta}-\frac{\omega^{2}}{\beta}\right)\left(-m^{2} b_{1}^{2}+2 m^{2} b_{2}^{2}+a_{1}^{2} m^{2}+6 a_{2} m^{2} a_{0}\right)+b\left(3 a_{1}^{2} a_{2}+3 a_{0} a_{2}^{2}-6 a_{1} b_{1} b_{2}+3 a_{2} b_{2}^{2}-3 b_{1}^{2} a_{2}-3 a_{0} b_{2}^{2}\right)=0, \\
& \left(\frac{k^{2} a^{2}}{\beta}-\frac{\omega^{2}}{\beta}\right)\left(2 a_{1} m^{2} b_{1}+6 m^{2} b_{2} a_{0}-a_{2} m^{2} b_{2}\right)+b\left(-3 b_{1}^{2} b_{2}+3 a_{1}^{2} b_{2}+b_{2}^{3}+6 a_{0} a_{2} b_{2}+6 a_{1} b_{1} a_{2}\right)=0, \\
& \left(\frac{k^{2} a^{2}}{\beta}-\frac{\omega^{2}}{\beta}\right)\left(b_{1} b_{2}-a_{1} a_{2}-a_{1} m^{2} a_{2}+2 a_{1} m^{2} a_{0}+7 m^{2} b_{1} b_{2}\right) \\
& +b\left(a_{1}^{3}-3 a_{1} b_{1}^{2}-6 a_{0} b_{1} b_{2}+6 a_{0} a_{1} a_{2}+3 a_{1} b_{2}^{2}+6 b_{1} a_{2} b_{2}\right)=0,
\end{aligned}
$$




$$
\begin{aligned}
& \left(\frac{k^{2} a^{2}}{\beta}-\frac{\omega^{2}}{\beta}\right)\left(-b_{1} a_{2}-a_{1} b_{2}+2 m^{2} b_{1} a_{0}-4 m^{2} b_{1} a_{2}\right)+b\left(3 a_{1}^{2} b_{1}-b_{1}^{3}+3 b_{1} b_{2}^{2}+6 a_{0} a_{1} b_{2}+6 a_{0} b_{1} a_{2}\right)=0, \\
& \left(\frac{k^{2} a^{2}}{\beta}-\frac{\omega^{2}}{\beta}\right)\left(-4 a_{2} a_{0}+2 m^{2} b_{1}^{2}-4 a_{2} m^{2} a_{0}-2 a_{2}^{2}\right)+b\left(3 a_{0} a_{1}^{2}-3 a_{0} b_{1}^{2}+3 a_{2} a_{0}^{2}+3 b_{1}^{2} a_{2}+3 a_{0} b_{2}^{2}+6 a_{1} b_{1} b_{2}\right)=0, \\
& \left(\frac{k^{2} a^{2}}{\beta}-\frac{\omega^{2}}{\beta}\right)\left(-2 a_{2} b_{2}-4 b_{2} a_{0}-m^{2} b_{2} a_{0}-a_{1} m^{2} b_{1}\right)+b\left(6 a_{0} a_{1} b_{1}+3 b_{2} a_{0}^{2}+3 b_{1}^{2} b_{2}\right)=0, \\
& \left(\frac{k^{2} a^{2}}{\beta}-\frac{\omega^{2}}{\beta}\right)\left(-a_{0} b_{1}+2 b_{1} a_{2}-2 a_{1} b_{2}\right)+b\left(3 a_{0}^{2} b_{1}+b_{1}^{3}\right)=0, \\
& \left(\frac{k^{2} a^{2}}{\beta}-\frac{\omega^{2}}{\beta}\right)\left(-a_{0} a_{1}-3 b_{1} b_{2}-2 a_{1} a_{2}-a_{1} m^{2} a_{0}-m^{2} b_{1} b_{2}\right)+b\left(3 a_{0}^{2} a_{1}+3 a_{1} b_{1}^{2}+6 a_{0} b_{1} b_{2}\right)=0, \\
& \left(\frac{k^{2} a^{2}}{\beta}-\frac{\omega^{2}}{\beta}\right)\left(-b_{2}^{2}-b_{1}^{2}+2 a_{2} a_{0}-a_{1}^{2}\right)+b\left(a_{0}^{3}+3 a_{0} b_{1}^{2}\right)=0
\end{aligned}
$$

Solving the above system with the aid of Maple or Mathematica, we have the following solution:

$$
a=a, b=\frac{-2\left(k^{2} a^{2}-\omega^{2}\right)}{\beta a_{2}}, k=k, m= \pm 1, \beta=\beta, a_{0}=-a_{2}, a_{1}=0, a_{2}=a_{2}, b_{1}=b_{2}=0 .
$$

So that the solve of Equation (36) can be written in the form

$$
\begin{gathered}
v=\frac{2\left(k^{2} a^{2}-\omega^{2}\right)}{\beta b}-\frac{2\left(k^{2} a^{2}-\omega^{2}\right)}{\beta b} s n^{2}, \\
u=\frac{1}{\beta} \ln \left(\frac{2\left(k^{2} a^{2}-\omega^{2}\right)}{\beta b}-\frac{2\left(k^{2} a^{2}-\omega^{2}\right)}{\beta b} s n^{2}\right) .
\end{gathered}
$$

When $m=1$, the solution can be in the form

$$
\begin{gathered}
v=\frac{2\left(k^{2} a^{2}-\omega^{2}\right)}{\beta b}-\frac{2\left(k^{2} a^{2}-\omega^{2}\right)}{\beta b} \tanh ^{2}(\xi), \\
u=\frac{1}{\beta} \ln \left(\frac{2\left(k^{2} a^{2}-\omega^{2}\right)}{\beta b}-\frac{2\left(k^{2} a^{2}-\omega^{2}\right)}{\beta b} \tanh ^{2}(\xi)\right) .
\end{gathered}
$$

\section{Conclusions}

We establish exact solutions for the system of shallow water wave equations and modified Liouville equation which are two of the most fascinating problems of modern mathematical physics.

The extended Jacobian elliptic function expansion method has been successfully used to find the exact traveling wave solutions of some nonlinear evolution equations. As an application, the traveling wave solutions for the system of shallow water wave equations and modified Liouville equation, have been constructed using the extended Jacobian elliptic function expansion method. Let us compare between our results obtained in the present article with the well-known results obtained by other authors using different methods as follows: our results of the system of shallow water wave equations and modified Liouville equation are new and different from those obtained in [27] and [28] and Figure 1 and Figure 2 show the solitary wave solution of Equations 


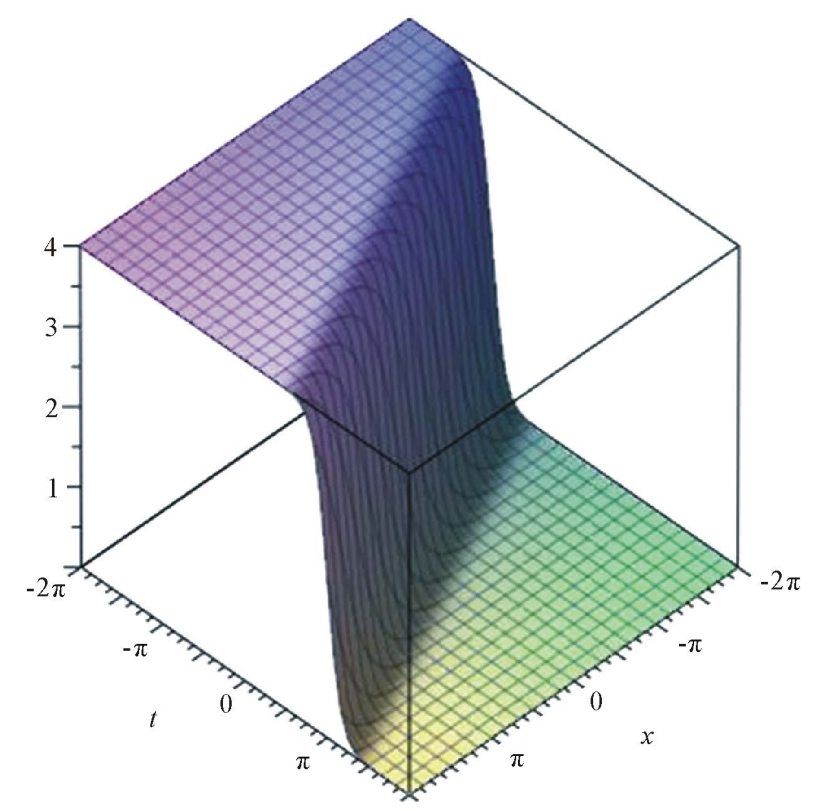

Figure 1. Solitary wave solution of Equation (30).

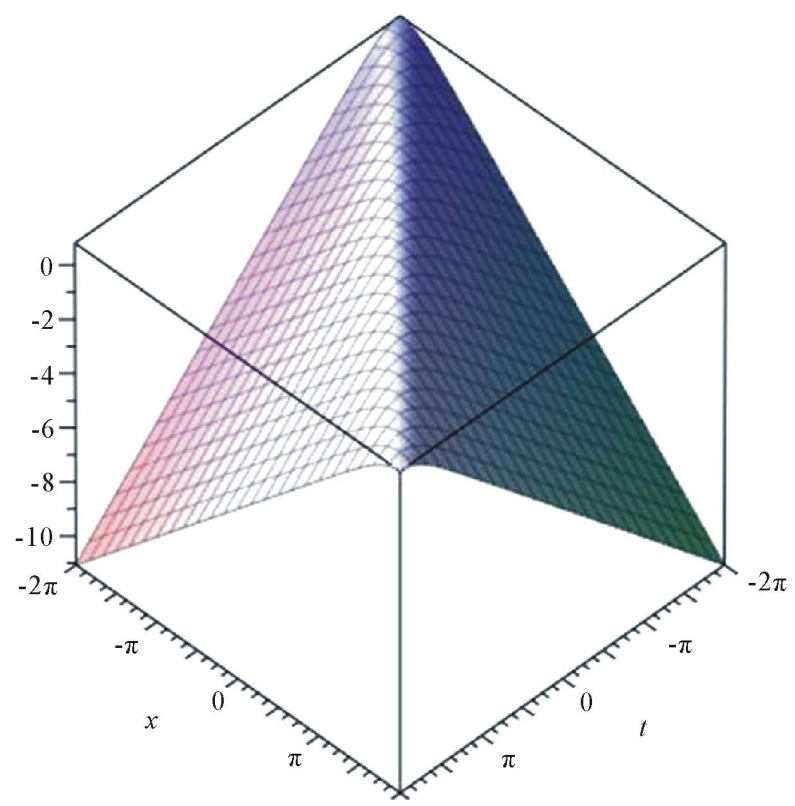

Figure 2. Solitary wave solution of Equation (56).

(30) and (56). It can be concluded that this method is reliable and proposes a variety of exact solutions NPDEs. The performance of this method is effective and can be applied to many other nonlinear evolution equations.

\section{References}

[1] Ablowitz, M.J. and Segur, H. (1981) Solitions and Inverse Scattering Transform. SIAM, Philadelphia. http://dx.doi.org/10.1137/1.9781611970883

[2] Malfliet, W. (1992) Solitary Wave Solutions of Nonlinear Wave Equation. American Journal of Physics, 60, 650-654. http://dx.doi.org/10.1119/1.17120

[3] Malfliet, W. and Hereman, W. (1996) The tanh Method: Exact Solutions of Nonlinear Evolution and Wave Equations. Physica Scripta, 54, 563-568. http://dx.doi.org/10.1088/0031-8949/54/6/003 
[4] Wazwaz, A.M. (2004) The tanh Method for Travelling Wave Solutions of Nonlinear Equations. Applied Mathematics and Computation, 154, 714-723. http://dx.doi.org/10.1016/S0096-3003(03)00745-8

[5] El-Wakil, S.A. and Abdou, M.A. (2007) New Exact Travelling Wave Solutions Using Modified Extented tanh-Function Method. Chaos, Solitons \& Fractals, 31, 840-852. http://dx.doi.org/10.1016/j.chaos.2005.10.032

[6] Fan, E. (2000) Extended tanh-Function Method and Its Applications to Nonlinear Equations. Physics Letters A, 277, 212-218. http://dx.doi.org/10.1016/S0375-9601(00)00725-8

[7] Wazwaz, A.M. (2007) The Extended tanh Method for Abundant Solitary Wave Solutions of Nonlinear Wave Equations. Applied Mathematics and Computation, 187, 1131-1142. http://dx.doi.org/10.1016/j.amc.2006.09.013

[8] Wazwaz, A.M. (2005) Exact Solutions to the Double sinh-Gordon Equation by the tanh Method and a Variable Separated ODE Method. Computers \& Mathematics with Applications, 50, 1685-1696. http://dx.doi.org/10.1016/j.camwa.2005.05.010

[9] Wazwaz, A.M. (2004) A sine-cosine Method for Handling Nonlinear Wave Equations. Mathematical and Computer Modelling, 40, 499-508. http://dx.doi.org/10.1016/j.mcm.2003.12.010

[10] Yan, C. (1996) A Simple Transformation for Nonlinear Waves. Physics Letters A, 224, 77-84. http://dx.doi.org/10.1016/S0375-9601(96)00770-0

[11] Fan, E. and Zhang, H. (1998) A Note on the Homogeneous Balance Method. Physics Letters A, 246, 403-406. http://dx.doi.org/10.1016/S0375-9601(98)00547-7

[12] Wang, M.L. (1996) Exact Solutions for a Compound KdV-Burgers Equation. Physics Letters A, 213, $279-287$. http://dx.doi.org/10.1016/0375-9601(96)00103-X

[13] Abdou, M.A. (2007) The Extended F-Expansion Method and Its Application for a Class of Nonlinear Evolution Equations. Chaos, Solitons \& Fractals, 31, 95-104. http://dx.doi.org/10.1016/j.chaos.2005.09.030

[14] Ren, Y.J. and Zhang, H.Q. (2006) A Generalized F-Expansion Method to Find Abundant Families of Jacobi Elliptic Function Solutions of the $(2+1)$-Dimensional Nizhnik-Novikov-Veselov Equation. Chaos, Solitons \& Fractals, 27, 959-979. http://dx.doi.org/10.1016/j.chaos.2005.04.063

[15] Zhang, J.L., Wang, M.L., Wang, Y.M. and Fang, Z.D. (2006) The Improved F-Expansion Method and Its Applications. Physics Letters A, 350, 103-109. http://dx.doi.org/10.1016/j.physleta.2005.10.099

[16] He, J.H. and Wu, X.H. (2006) Exp-Function Method for Nonlinear Wave Equations. Chaos, Solitons \& Fractals, 30, 700-708. http://dx.doi.org/10.1016/j.chaos.2006.03.020

[17] Zahran, E.H.M. and Khater, M.M.A. (2014) The Modified Simple Equation Method and Its Applications for Solving Some Nonlinear Evolutions Equations in Mathematical Physics. Jokull Journal, 64.

[18] Abdelrahman, M.A.E., Zahran, E.H.M. and Khater, M.M.A. (2014) Exact Traveling Wave Solutions for Power Law and Kerr Law Non Linearity Using the $\exp (-\phi(\xi))$-Expansion Method. Global Journal of Science Frontier Research, 14-F.

[19] Wang, M.L., Zhang, J.L. and Li, X.Z. (2008) The $\left(\frac{G^{\prime}}{G}\right)$-Expansion Method and Travelling Wave Solutions of Nonlinear Evolutions Equations in Mathematical Physics. Physics Letters A, 372, 417-423. http://dx.doi.org/10.1016/j.physleta.2007.07.051

[20] Zhang, S., Tong, J.L. and Wang, W. (2008) A Generalized $\left(\frac{G^{\prime}}{G}\right)$-Expansion Method for the mKdv Equation with Variable Coefficients. Physics Letters A, 372, 2254-2257. http://dx.doi.org/10.1016/j.physleta.2007.11.026

[21] Zayed, E.M.E. and Gepreel, K.A. (2009) The $\left(\frac{G^{\prime}}{G}\right)$-Expansion Method for Finding Traveling Wave Solutions of Nonlinear Partial Differential Equations in Mathematical Physics. Journal of Mathematical Physics, 50, Article ID: 013502. http://dx.doi.org/10.1063/1.3033750

[22] Zahran, E.H.M. and Khater, M.M.A. (2014) Exact Solution to Some Nonlinear Evolution Equations by the $\left(\frac{G^{\prime}}{G}\right)$-Expansion Method. Jokull Journal, 64.

[23] Zaki, S.I. (2000) Solitary Wave Interactions for the Modified Equal width Wave Equation. Computer Physics Communications, 126, 219-213. http://dx.doi.org/10.1016/S0010-4655(99)00471-3

[24] Fan, E. and Zhang, J. (2002) Applications of the Jacobi Elliptic Function Method to Special-Type Nonlinear Equations. Physics Letters A, 305, 383-392. http://dx.doi.org/10.1016/S0375-9601(02)01516-5 
[25] Liu, S., Fu, Z., Liu, S. and Zhao, Q. (2001) Jacobi Elliptic Function Expansion Method and Periodic Wave Solutions of Nonlinear Wave Equations. Physics Letters A, 289, 69-74. http://dx.doi.org/10.1016/S0375-9601(01)00580-1

[26] Zhao, X.Q., Zhi, H.Y. and Zhang, H.Q. (2006) Improved Jacobi-Function Method with Symbolic Computation to Construct New Double-Periodic Solutions for the Generalized Ito System. Chaos, Solitons \& Fractals, 28, 112-126. http://dx.doi.org/10.1016/j.chaos.2005.05.016

[27] Dolapci, İ.T. and Yildirim, A. (2013) Some Exact Solutions to the Generalized Korteweg-de Vries Equation and the System of Shallow Water Wave Equation. Nonlinear Analysis, Modeling and Control, 18, 27-36.

[28] Salam, M.A. (2012) Traveling-Wave Solution of Modified Liouville Equation by Means of Modified Simple Equation Method. International Scholarly Research Network ISRN Applied Mathematics, 2012, Article ID: 565247. 
Scientific Research Publishing (SCIRP) is one of the largest Open Access journal publishers. It is currently publishing more than 200 open access, online, peer-reviewed journals covering a wide range of academic disciplines. SCIRP serves the worldwide academic communities and contributes to the progress and application of science with its publication.

Other selected journals from SCIRP are listed as below. Submit your manuscript to us via either submit@scirp.org or Online Submission Portal.
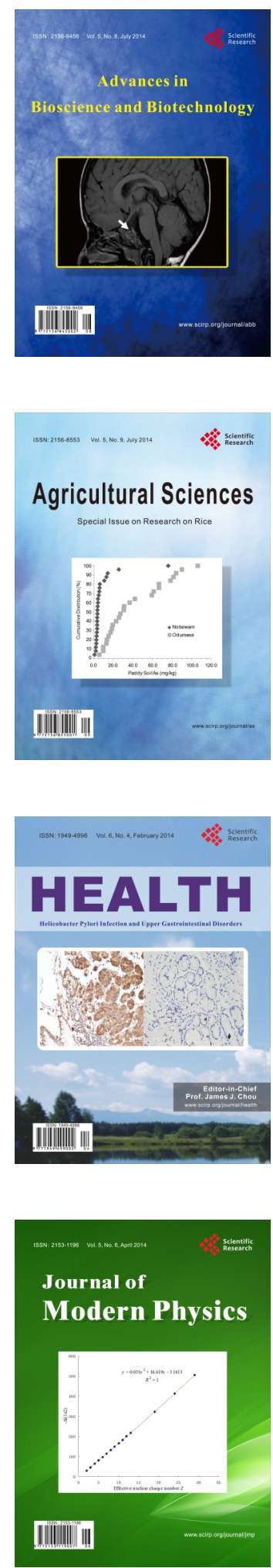
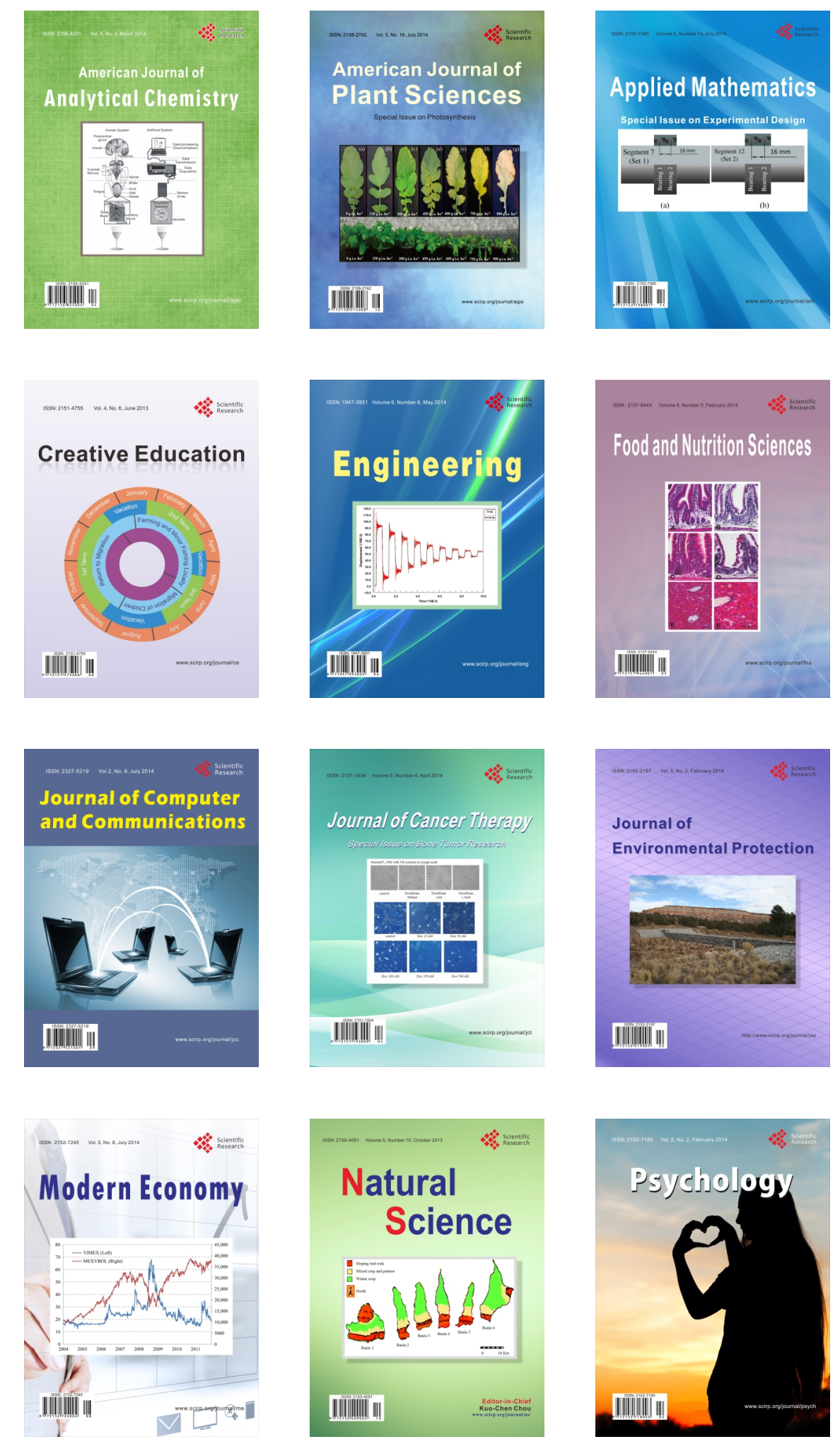